\title{
Stabilization of a Flexible Cable with Two Rigid Loads
}

\author{
Hideki SANO** and Masashi OtAnAKA* \\ ** Computing and Communications Center, Kagoshima University, \\ 1-21-35 Korimoto, Kagoshima 890-0065, Japan \\ E-mail: sano@cc.kagoshima-u.ac.jp \\ * Graduate School of Science and Engineering, Kagoshima University, \\ 1-21-35 Korimoto, Kagoshima 890-0065, Japan
}

Received September 9, 2005

Revised May 10, 2006

\begin{abstract}
In this paper, we study the stabilization problem of a flexible cable with two rigid loads which is described by two kinds of hyperbolic equations. From the engineering point of view, the model can be regarded as a distributed parameter overhead crane which conveys two loads simultaneously. After deriving a control law which does not increase an energy defined for the model, we prove the asymptotic stability of the closed-loop system, using the LaSalle's invariance principle. Finally, we show that the control law works effectively through a numerical simulation.
\end{abstract}

Key words: flexible cable, $C_{0}$-semigroup, asymptotic stability, LaSalle's invariance principle

\section{Introduction}

The stabilization and optimal control of strings without natural damping are challenging topics in the field of distributed parameter systems. Since old times, several types of problems in this direction have been investigated by many researchers (see, for example, [1], [2], [3], [5, Chapter 6], [8, Chapter 6], [10], and the references therein). Especially, from the practical point of view, Rao has treated the stabilization problem of suppressing the vibration of a distributed parameter overhead crane model with one rigid load [10]. In that paper, after deriving a control law, the energy multiplier method is applied to the closed-loop system and the exponential stability of the energy is proved. Also, d'Andréa-Novel and Coron have proposed a back-stepping approach for the similar problem [2].

On the other hand, in the field of lumped parameter systems, the problem of stabilizing the double-pendulum system has been widely investigated from both theoretical and experimental aspects. However, as far as the authors know, a distributed parameter model for it, which may be regarded as a system subjected to distributed perturbation such as wind, has not been treated. Therefore, we study, in this paper, the stabilization problem of a flexible cable with two rigid loads which is described by two kinds of hyperbolic equations. From the engineering point of view, the model can be regarded as a distributed parameter overhead crane which conveys two loads simultaneously. Our aim is to suppress the vibration of the two loads as well as the cable, using a control law. 
In this paper, we first define an energy for the flexible cable with two rigid loads and derive a control law by using the similar way as in [10]. However, it seems difficult to use the energy multiplier method to show the exponential stability of the energy for the closed-loop system consisting of the cable with two rigid loads and the control law, because the model is complicated compared with Rao's one. So, we concentrate on the proof of the asymptotic stability of the closed-loop system which is based on the LaSalle's invariance principle. Moreover, we show that the control law works effectively through a numerical simulation. In this paper, two Hilbert spaces $X$ and $W$ will be introduced, where $X$ is a Hilbert space on which the closed-loop operator generates a $C_{0}$-semigroup and $W$ is another Hilbert space such that the embedding of $W$ in $X$ is compact. The introduction of the Hilbert space $W$ will play an important role in the proof of Lemma 1.

\section{System Description}

Let $b$ be a constant such that $0<b<1$. We shall consider the following flexible cable with two rigid loads:

$$
\begin{cases}\frac{\partial^{2} y}{\partial t^{2}}(t, x)=\frac{\partial}{\partial x}\left(a(x) \frac{\partial y}{\partial x}(t, x)\right), & t>0, x \in(0, b), \\ \frac{\partial^{2} y}{\partial t^{2}}(t, x)=\frac{\partial}{\partial x}\left(\tilde{a}(x) \frac{\partial y}{\partial x}(t, x)\right), & t>0, x \in(b, 1), \\ a(0) \frac{\partial y}{\partial x}(t, 0)=u(t), & t>0, \\ y\left(t, b^{-}\right)=y\left(t, b^{+}\right), & t \geq 0, \\ M_{1} \frac{\partial^{2} y}{\partial t^{2}}(t, b)=\tilde{a}\left(b^{+}\right) \frac{\partial y}{\partial x}\left(t, b^{+}\right)-a\left(b^{-}\right) \frac{\partial y}{\partial x}\left(t, b^{-}\right), & t>0, \\ M_{2} \frac{\partial^{2} y}{\partial t^{2}}(t, 1)=-\tilde{a}(1) \frac{\partial y}{\partial x}(t, 1), & t>0, \\ y(0, x)=p(x), \quad \frac{\partial y}{\partial t}(0, x)=q(x), & x \in(0,1) .\end{cases}
$$

In the above, $u(t)$ denotes the control force, and $a(x):=g\left(M_{1}+M_{2}+1-x\right)(0 \leq$ $x<b)$ and $\tilde{a}(x):=g\left(M_{2}+1-x\right)(b<x \leq 1)$ the tension force of the cable at the point $x$, where $g$ is the gravitational acceleration, and $M_{1}$ and $M_{2}$ the masses of rigid loads. As shown in Fig. 1, system (2.1) expresses a distributed parameter overhead crane model with two rigid loads. Here, it is assumed that each load is a mass point and that the mass of the cart, which is sufficiently small compared with the one of each load, is neglected. Moreover, it is supposed that the displacement $y(t, x)$ and its derivative $y_{x}(t, x)$ are small through the cable and that the total mass of the cable is 1 , i.e., the line density is equal to 1 .

REMARK 1. See [10] for a distributed parameter overhead crane model with one rigid load. 


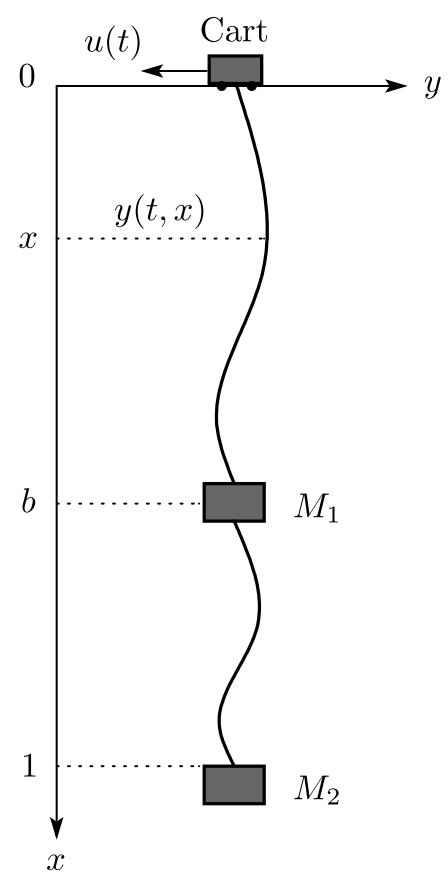

Fig. 1. Distributed parameter overhead crane model with two rigid loads.

\section{Derivation of Control Law}

Let us define the following energy $E(t)$ for system (2.1):

$$
\begin{aligned}
E(t):=\frac{1}{2}[ & \int_{0}^{b}\left\{a(x) y_{x}^{2}(t, x)+y_{t}^{2}(t, x)\right\} d x+\int_{b}^{1}\left\{\tilde{a}(x) y_{x}^{2}(t, x)+y_{t}^{2}(t, x)\right\} d x \\
& \left.+\alpha y^{2}(t, 0)+M_{1} y_{t}^{2}(t, b)+M_{2} y_{t}^{2}(t, 1)\right], \quad \alpha>0 .
\end{aligned}
$$

Differentiating (3.1) with respect to $t$, and using (2.1) and integration by parts yields

$$
\begin{aligned}
\frac{d}{d t} E(t)= & \int_{0}^{b}\left\{a(x) y_{x}(t, x) y_{x t}(t, x)+y_{t}(t, x) y_{t t}(t, x)\right\} d x \\
& +\int_{b}^{1}\left\{\tilde{a}(x) y_{x}(t, x) y_{x t}(t, x)+y_{t}(t, x) y_{t t}(t, x)\right\} d x+\alpha y(t, 0) y_{t}(t, 0) \\
& +M_{1} y_{t}(t, b) y_{t t}(t, b)+M_{2} y_{t}(t, 1) y_{t t}(t, 1) \\
= & -y_{t}(t, 0)(u(t)-\alpha y(t, 0))
\end{aligned}
$$


Here, choosing the control input $u(t)$ as

$$
u(t)=\alpha y(t, 0)+\gamma y_{t}(t, 0), \quad \gamma>0,
$$

we have

$$
\frac{d}{d t} E(t)=-\gamma y_{t}^{2}(t, 0) \leq 0
$$

Consequently, the energy $E(t)$ for system (2.1) becomes nonincreasing under the control law (3.2).

\section{Closed-Loop System}

The closed-loop system consisting of (2.1) and (3.2) is described by

$$
\begin{cases}y_{t t}(t, x)=\left(a(x) y_{x}(t, x)\right)_{x}, & t>0, x \in(0, b), \\ y_{t t}(t, x)=\left(\tilde{a}(x) y_{x}(t, x)\right)_{x}, & t>0, x \in(b, 1), \\ a(0) y_{x}(t, 0)=\alpha y(t, 0)+\gamma y_{t}(t, 0), & t>0, \\ y\left(t, b^{-}\right)=y\left(t, b^{+}\right), & t \geq 0, \\ M_{1} y_{t t}(t, b)=\tilde{a}\left(b^{+}\right) y_{x}\left(t, b^{+}\right)-a\left(b^{-}\right) y_{x}\left(t, b^{-}\right), & t>0, \\ M_{2} y_{t t}(t, 1)=-\tilde{a}(1) y_{x}(t, 1), & t>0, \\ y(0, x)=p(x), \quad y_{t}(0, x)=q(x), & x \in(0,1) .\end{cases}
$$

In order to formulate this closed-loop system in an abstract space, let us introduce the Hilbert space

$X=\left\{\left[\begin{array}{c}y(\cdot) \\ z(\cdot) \\ \tilde{y}(\cdot) \\ \tilde{z}(\cdot) \\ \xi \\ \eta\end{array}\right] \in H^{1}(0, b) \times L^{2}(0, b) \times H^{1}(b, 1) \times L^{2}(b, 1) \times \mathbf{R} \times \mathbf{R} ; y(b)=\tilde{y}(b)\right\}$

with the inner product

$$
\begin{aligned}
\left\langle f_{1}, f_{2}\right\rangle_{X}= & \int_{0}^{b}\left\{a(x) y_{1 x}(x) y_{2 x}(x)+z_{1}(x) z_{2}(x)\right\} d x \\
& +\int_{b}^{1}\left\{\tilde{a}(x) \tilde{y}_{1 x}(x) \tilde{y}_{2 x}(x)+\tilde{z}_{1}(x) \tilde{z}_{2}(x)\right\} d x \\
& +\alpha y_{1}(0) y_{2}(0)+M_{1} \xi_{1} \xi_{2}+M_{2} \eta_{1} \eta_{2},
\end{aligned}
$$

for $f_{1}=\left[y_{1}, z_{1}, \tilde{y}_{1}, \tilde{z}_{1}, \xi_{1}, \eta_{1}\right]^{T} \in X, \quad f_{2}=\left[y_{2}, z_{2}, \tilde{y}_{2}, \tilde{z}_{2}, \xi_{2}, \eta_{2}\right]^{T} \in X$. 
In the above, $H^{1}(0, b)$ and $H^{1}(b, 1)$ are the usual Sobolev spaces. Here, we define the operator $A: D(A) \subset X \rightarrow X$ as follows:

$$
\begin{aligned}
A & {\left[\begin{array}{c}
y(\cdot) \\
z(\cdot) \\
\tilde{y}(\cdot) \\
\tilde{z}(\cdot) \\
\xi \\
\eta
\end{array}\right]=\left[\begin{array}{c}
-z(\cdot) \\
-\left(a(\cdot) y_{x}(\cdot)\right)_{x} \\
-\tilde{z}(\cdot) \\
-\left(\tilde{a}(\cdot) \tilde{y}_{x}(\cdot)\right)_{x} \\
-\frac{\tilde{a}\left(b^{+}\right) \tilde{y}_{x}\left(b^{+}\right)-a\left(b^{-}\right) y_{x}\left(b^{-}\right)}{M_{1}} \\
\frac{\tilde{a}(1) \tilde{y}_{x}(1)}{M_{2}}
\end{array}\right],\left[\begin{array}{c}
y(\cdot) \\
z(\cdot) \\
\tilde{y}(\cdot) \\
\tilde{z}(\cdot) \\
\xi \\
\eta
\end{array}\right] \in D(A), } \\
D(A)= & \left\{\begin{array}{c}
{\left[\begin{array}{c}
y(\cdot) \\
z(\cdot) \\
\tilde{y}(\cdot) \\
\tilde{z}(\cdot) \\
\xi \\
\eta
\end{array}\right] \in H^{2}(0, b) \times H^{1}(0, b) \times H^{2}(b, 1) \times H^{1}(b, 1) \times \mathbf{R} \times \mathbf{R} ;} \\
\xi=z(b)=\tilde{z}(b), \eta=\tilde{z}(1), a(0) y_{x}(0)=\alpha y(0)+\gamma z(0), y(b)=\tilde{y}(b)
\end{array}\right\} .
\end{aligned}
$$

Denoting $y(t, x)$ restricted to $0 \leq x \leq b$ by $y(t, x)$, and $y(t, x)$ restricted to $b<$ $x \leq 1$ by $\tilde{y}(t, x)$, and moreover introducing the new variables $z(t, \cdot)=y_{t}(t, \cdot)$, $\tilde{z}(t, \cdot)=\tilde{y}_{t}(t, \cdot), \xi(t)=y_{t}(t, b)\left(=y_{t}\left(t, b^{-}\right)=\tilde{y}_{t}\left(t, b^{+}\right)\right)$, and $\eta(t)=\tilde{y}_{t}(t, 1)$, the closed-loop system (4.1) can be written as

$$
\frac{d}{d t} f(t)=-A f(t)
$$

where $f(t):=[y(t, \cdot), z(t, \cdot), \tilde{y}(t, \cdot), \tilde{z}(t, \cdot), \xi(t), \eta(t)]^{T}$. If the operator $-A$ generates a $C_{0}$-semigroup $e^{-t A}$ on $X$, then the solution of (4.2) is expressed as

$$
f(t)=e^{-t A} f(0)
$$

\section{Closed-Loop Stability}

In this section, we prove that the closed-loop system (4.2) is asymptotically stable by using the LaSalle's invariance principle. As the first step, we show that the operator $-A$ generates a $C_{0}$-semigroup of contractions $e^{-t A}$ on $X$. Then, we show that the operator $(I+\lambda A)^{-1}$ is compact for every $\lambda>0$.

THEOREM 1. The operator $-A$ generates a $C_{0}$-semigroup of contractions $e^{-t A}$ on $X$. 
Proof. By the definitions of the inner product of $X$ and the operator $A$, we can calculate $\langle-A f, f\rangle_{X}$ as follows:

$$
\langle-A f, f\rangle_{X}=-\gamma z^{2}(0) \leq 0, \quad \forall f=[y, z, \tilde{y}, \tilde{z}, \xi, \eta]^{T} \in D(A)
$$

which implies that $-A$ is dissipative. Next, we shall discuss whether, for each $f_{0}=\left[y_{0}, z_{0}, \tilde{y}_{0}, \tilde{z}_{0}, \xi_{0}, \eta_{0}\right]^{T} \in X$, there exists a unique $f=[y, z, \tilde{y}, \tilde{z}, \xi, \eta]^{T} \in D(A)$ such that

$$
(I+A) f=f_{0}
$$

Eliminating $z$ in equation (5.1), we have

$$
\begin{aligned}
& y(\cdot)-\left(a(\cdot) y_{x}(\cdot)\right)_{x}=y_{0}(\cdot)+z_{0}(\cdot), \\
& \tilde{y}(\cdot)-\left(\tilde{a}(\cdot) \tilde{y}_{x}(\cdot)\right)_{x}=\tilde{y}_{0}(\cdot)+\tilde{z}_{0}(\cdot), \\
& y(b)-y_{0}(b)-\frac{\tilde{a}\left(b^{+}\right) \tilde{y}_{x}\left(b^{+}\right)-a\left(b^{-}\right) y_{x}\left(b^{-}\right)}{M_{1}}=\xi_{0}, \\
& \tilde{y}(b)-\tilde{y}_{0}(b)-\frac{\tilde{a}\left(b^{+}\right) \tilde{y}_{x}\left(b^{+}\right)-a\left(b^{-}\right) y_{x}\left(b^{-}\right)}{M_{1}}=\xi_{0}, \\
& \tilde{a}(1) \tilde{y}_{x}(1)+M_{2} \tilde{y}(1)=M_{2}\left(\tilde{y}_{0}(1)+\eta_{0}\right), \\
& a(0) y_{x}(0)-(\alpha+\gamma) y(0)=-\gamma y_{0}(0) .
\end{aligned}
$$

For equation (5.2), there exists a unique solution $y(\cdot) \in H^{2}(0, b)$, since $y_{0}(\cdot)+$ $z_{0}(\cdot) \in L^{2}(0, b)$. Similarly, for equation (5.3), there exists a unique solution $\tilde{y}(\cdot) \in$ $H^{2}(b, 1)$, since $\tilde{y}_{0}(\cdot)+\tilde{z}_{0}(\cdot) \in L^{2}(b, 1)$. Here, defining $z(\cdot):=y(\cdot)-y_{0}(\cdot)$ and $\tilde{z}(\cdot):=\tilde{y}(\cdot)-\tilde{y}_{0}(\cdot)$, it follows that $z(\cdot) \in H^{1}(0, b)$ and $\tilde{z}(\cdot) \in H^{1}(b, 1)$, and that

$$
z(\cdot)-\left(a(\cdot) y_{x}(\cdot)\right)_{x}=z_{0}(\cdot), \quad \tilde{z}(\cdot)-\left(\tilde{a}(\cdot) \tilde{y}_{x}(\cdot)\right)_{x}=\tilde{z}_{0}(\cdot) .
$$

Further, from (5.4)-(5.7), we get

$$
\begin{aligned}
& z(b)-\frac{\tilde{a}\left(b^{+}\right) \tilde{y}_{x}\left(b^{+}\right)-a\left(b^{-}\right) y_{x}\left(b^{-}\right)}{M_{1}}=\xi_{0}, \\
& \tilde{z}(b)-\frac{\tilde{a}\left(b^{+}\right) \tilde{y}_{x}\left(b^{+}\right)-a\left(b^{-}\right) y_{x}\left(b^{-}\right)}{M_{1}}=\xi_{0}, \\
& \tilde{z}(1)+\frac{\tilde{a}(1) \tilde{y}_{x}(1)}{M_{2}}=\eta_{0}, \quad a(0) y_{x}(0)=\alpha y(0)+\gamma z(0),
\end{aligned}
$$

respectively. From this, we have $y(b)=\tilde{y}(b)$ since $y_{0}(b)=\tilde{y}_{0}(b)$. Hence, $[y(\cdot)$, $z(\cdot), \tilde{y}(\cdot), \tilde{z}(\cdot), z(b), \tilde{z}(1)]^{T}=[y(\cdot), z(\cdot), \tilde{y}(\cdot), \tilde{z}(\cdot), \tilde{z}(b), \tilde{z}(1)]^{T} \in D(A)$ solves equation (5.1). In this way, we see that, for each $f_{0} \in X$, there exists a unique $f \in D(A)$ that satisfies (5.1). Also, it is not difficult to show that the domain $D(A)$ is dense in $X$. Consequently, by the Lumer-Phillips' theorem [9, Theorem 1.4.3], the operator $-A$ generates a $C_{0}$-semigroup of contractions $e^{-t A}$ on $X$. 
Now, let us introduce another Hilbert space

$$
W=\left\{\begin{array}{c}
y(\cdot) \\
z(\cdot) \\
\tilde{y}(\cdot) \\
\tilde{z}(\cdot) \\
\xi \\
\eta
\end{array}\right] \in H^{2}(0, b) \times H^{1}(0, b) \times H^{2}(b, 1) \times H^{1}(b, 1) \times \mathbf{R} \times \mathbf{R} ;
$$

$$
\xi=z(b)=\tilde{z}(b), \quad y(b)=\tilde{y}(b)\}
$$

with the inner product

$$
\begin{gathered}
\begin{array}{c}
\left\langle f_{1}, f_{2}\right\rangle_{W}=\left\langle y_{1}, y_{2}\right\rangle_{H^{2}(0, b)}+\left\langle z_{1 x}, z_{2 x}\right\rangle_{L^{2}(0, b)}+\left\langle\tilde{y}_{1}, \tilde{y}_{2}\right\rangle_{H^{2}(b, 1)}+\left\langle\tilde{z}_{1 x}, \tilde{z}_{2 x}\right\rangle_{L^{2}(b, 1)} \\
\\
+\xi_{1} \xi_{2}+\eta_{1} \eta_{2}+z_{1}(0) z_{2}(0),
\end{array} \\
\text { for } \quad f_{1}=\left[y_{1}, z_{1}, \tilde{y}_{1}, \tilde{z}_{1}, \xi_{1}, \eta_{1}\right]^{T} \in W, \quad f_{2}=\left[y_{2}, z_{2}, \tilde{y}_{2}, \tilde{z}_{2}, \xi_{2}, \eta_{2}\right]^{T} \in W .
\end{gathered}
$$

Then, the inclusion relation $D(A) \subset W \subset X$ clearly holds, and it is not difficult to show that the injection from $W$ into $X$ is continuous, i.e., there exists a positive constant $C$ such that $\|f\|_{X} \leq C\|f\|_{W}, \forall f \in W$. The Hilbert space $W$ will be used to prove the following Lemma 1 . Hereafter, the notation $C_{i}$ denotes a positive constant.

Lemma 1. For every $\lambda>0$, the operator $(I+\lambda A)^{-1}: X \rightarrow X$ is compact.

Proof. First, for given $f_{0}=\left[y_{0}, z_{0}, \tilde{y}_{0}, \tilde{z}_{0}, \xi_{0}, \eta_{0}\right]^{T} \in X$, we solve $f=[y, z, \tilde{y}, \tilde{z}$, $\xi, \eta]^{T} \in D(A)$ such that

$$
A f=f_{0} .
$$

From (5.8), $f=[y, z, \tilde{y}, \tilde{z}, \xi, \eta]^{T}$ is concretely calculated as

$$
\begin{aligned}
& z(\cdot)=-y_{0}(\cdot), \\
& \tilde{z}(\cdot)=-\tilde{y}_{0}(\cdot), \\
& \xi=-y_{0}(b)=-\tilde{y}_{0}(b), \\
& \eta=-\tilde{y}_{0}(1), \\
& y(\cdot)=y(0)+\int_{0}^{\cdot} \frac{1}{a(r)}\left\{\alpha y(0)-\gamma y_{0}(0)-\int_{0}^{r} z_{0}(s) d s\right\} d r, \\
& \tilde{y}(\cdot)=\tilde{y}(b)+\int_{b}^{\cdot} \frac{1}{\tilde{a}(r)}\left\{M_{2} \eta_{0}+\int_{b}^{1} \tilde{z}_{0}(s) d s-\int_{b}^{r} \tilde{z}_{0}(s) d s\right\} d r,
\end{aligned}
$$


where

$$
\begin{aligned}
& y(0)=\frac{1}{\alpha}\left\{\gamma y_{0}(0)+M_{1} \xi_{0}+M_{2} \eta_{0}+\int_{0}^{b} z_{0}(s) d s+\int_{b}^{1} \tilde{z}_{0}(s) d s\right\}, \\
& \tilde{y}(b)=y(b)=y(0)+\int_{0}^{b} \frac{1}{a(r)}\left\{\alpha y(0)-\gamma y_{0}(0)-\int_{0}^{r} z_{0}(s) d s\right\} d r .
\end{aligned}
$$

Therefore, the inverse operator $A^{-1}$ exists and $f$ is expressed as $f=A^{-1} f_{0}$.

Next, we shall estimate the $W$-norm bound of $f=A^{-1} f_{0}$. From (5.15) and (5.16), we have

$$
\begin{aligned}
& |y(0)| \leq C_{1}\left\{\left|y_{0}(0)\right|+\left|\xi_{0}\right|+\left|\eta_{0}\right|+\left\|z_{0}\right\|_{L^{2}(0, b)}+\left\|\tilde{z}_{0}\right\|_{L^{2}(b, 1)}\right\} \\
& |\tilde{y}(b)|=|y(b)| \leq C_{2}\left\{\left|y_{0}(0)\right|+\left|\xi_{0}\right|+\left|\eta_{0}\right|+\left\|z_{0}\right\|_{L^{2}(0, b)}+\left\|\tilde{z}_{0}\right\|_{L^{2}(b, 1)}\right\}
\end{aligned}
$$

by using Hölder's inequality. Based on (5.17) and (5.18), we get the following $H^{2}$-norm estimates for (5.13) and (5.14):

$$
\begin{aligned}
\|y\|_{H^{2}(0, b)}^{2} & =\|y\|_{L^{2}(0, b)}^{2}+\left\|y_{x}\right\|_{L^{2}(0, b)}^{2}+\left\|y_{x x}\right\|_{L^{2}(0, b)}^{2} \\
& \leq C_{3}\left\{y_{0}^{2}(0)+\xi_{0}^{2}+\eta_{0}^{2}+\left\|z_{0}\right\|_{L^{2}(0, b)}^{2}+\left\|\tilde{z}_{0}\right\|_{L^{2}(b, 1)}^{2}\right\}, \\
\|\tilde{y}\|_{H^{2}(b, 1)}^{2} & =\|\tilde{y}\|_{L^{2}(b, 1)}^{2}+\left\|\tilde{y}_{x}\right\|_{L^{2}(b, 1)}^{2}+\left\|\tilde{y}_{x x}\right\|_{L^{2}(b, 1)}^{2} \\
& \leq C_{4}\left\{y_{0}^{2}(0)+\xi_{0}^{2}+\eta_{0}^{2}+\left\|z_{0}\right\|_{L^{2}(0, b)}^{2}+\left\|\tilde{z}_{0}\right\|_{L^{2}(b, 1)}^{2}\right\} .
\end{aligned}
$$

Also, from (5.11) and (5.12), we have

$$
\begin{aligned}
& |\xi| \leq\left|y_{0}(0)\right|+\left\|y_{0 x}\right\|_{L^{2}(0, b)}, \\
& |\eta| \leq\left|y_{0}(0)\right|+\left\|y_{0 x}\right\|_{L^{2}(0, b)}+\left\|\tilde{y}_{0 x}\right\|_{L^{2}(b, 1)},
\end{aligned}
$$

since $y_{0} \in H^{1}(0, b)$ and $\tilde{y}_{0} \in H^{1}(b, 1)$. In the above, we also used Hölder's inequality. Here, using (5.9), (5.10), and (5.19)-(5.22), we get

$$
\begin{aligned}
\left\|A^{-1} f_{0}\right\|_{W}^{2}= & \|f\|_{W}^{2} \\
= & \|y\|_{H^{2}(0, b)}^{2}+\left\|z_{x}\right\|_{L^{2}(0, b)}^{2}+\|\tilde{y}\|_{H^{2}(b, 1)}^{2}+\left\|\tilde{z}_{x}\right\|_{L^{2}(b, 1)}^{2}+\xi^{2}+\eta^{2}+z^{2}(0) \\
\leq & C_{5}\left\{y_{0}^{2}(0)+\xi_{0}^{2}+\eta_{0}^{2}\right. \\
& \left.\quad+\left\|z_{0}\right\|_{L^{2}(0, b)}^{2}+\left\|\tilde{z}_{0}\right\|_{L^{2}(b, 1)}^{2}+\left\|y_{0 x}\right\|_{L^{2}(0, b)}^{2}+\left\|\tilde{y}_{0 x}\right\|_{L^{2}(b, 1)}^{2}\right\} .
\end{aligned}
$$

On the other hand, it follows from the definition of the inner product of $X$ that

$$
\begin{aligned}
\left\|f_{0}\right\|_{X}^{2}=\left\langle f_{0}, f_{0}\right\rangle_{X} \\
\geq C_{6}\left\{y_{0}^{2}(0)+\xi_{0}^{2}+\eta_{0}^{2}\right. \\
\left.\quad+\left\|z_{0}\right\|_{L^{2}(0, b)}^{2}+\left\|\tilde{z}_{0}\right\|_{L^{2}(b, 1)}^{2}+\left\|y_{0 x}\right\|_{L^{2}(0, b)}^{2}+\left\|\tilde{y}_{0 x}\right\|_{L^{2}(b, 1)}^{2}\right\} .
\end{aligned}
$$


Combining (5.23) and (5.24), we obatin

$$
\left\|A^{-1} f_{0}\right\|_{W} \leq C_{7}\left\|f_{0}\right\|_{X}, \quad \forall f_{0} \in X
$$

From the Sobolev's embedding theorem, it follows that the embedding of $H^{1}(\Omega)$ in $L^{2}(\Omega)$ and the one of $H^{2}(\Omega)$ in $H^{1}(\Omega)$ are compact, $\Omega$ being $(0, b)$ or $(b, 1)$, which implies that the embedding of $W$ in $X$ is compact. From this fact and (5.25), we see that $A^{-1}$ is a compact operator from $X$ into itself. Moreover, noting that $\rho(-A) \supset(0, \infty)$ holds since $-A$ generates a $C_{0}$-semigroup of contractions $e^{-t A}$ on $X, \rho(-A)$ being the resolvent set of $-A$, we can conclude that $(I+\lambda A)^{-1}: X \rightarrow X$ is compact for every $\lambda>0$.

The following theorem is our main result in this paper.

TheOREM 2. The closed-loop system (4.2) is asymptotically stable, that is, for every $f(0) \in X,\left\|e^{-t A} f(0)\right\|_{X} \rightarrow 0$ as $t \rightarrow \infty$.

Proof. Let $\omega$ be the largest invariant subset of $\{f \in X ; d E(t) / d t=0\}$. If $\omega=\{0\}$, then we can conclude that, for every $f(0) \in X$,

$$
\left\|e^{-t A} f(0)\right\|_{X} \rightarrow 0 \quad \text { as } \quad t \rightarrow \infty
$$

by the LaSalle's invariance principle [8, Theorems 3.64 and 3.65], since we have already shown that Theorem 1 and Lemma 1 hold. In Section 3, it was also shown that $d E(t) / d t=-\gamma y_{t}^{2}(t, 0)$. Hence, in order to show $\omega=\{0\}$, we have only to prove that system (4.1) with the condition $y_{t}(t, 0)=0$ implies $y(t, x)=0$.

First, let us consider the subsystem

$$
\begin{cases}y_{t t}(t, x)=\left(a(x) y_{x}(t, x)\right)_{x}, & t>0, x \in(0, b), \\ a(0) y_{x}(t, 0)=\alpha y(t, 0)+\gamma y_{t}(t, 0), & t>0, \\ y_{t}(t, 0)=0, & t \geq 0 .\end{cases}
$$

From the second and third equations of (5.27), we have

$$
a(0) y_{x}(t, 0)=\alpha y(t, 0)
$$

Here, differentiating this equation with respect to $t$ yields

$$
a(0) y_{x t}(t, 0)=\alpha y_{t}(t, 0)=0,
$$

which implies that $y_{x t}(t, 0)=0$ since $a(0)>0$. Now, let us set $y(t, x)=T(t) \Phi(x)$. Then, it follows that $\Phi(0)=\Phi_{x}(0)=0$, and from the first equation of $(5.27)$ that

$$
\frac{T_{t t}(t)}{T(t)}=\frac{a(x) \Phi_{x x}(x)-g \Phi_{x}(x)}{\Phi(x)}=k,
$$


$k$ being a constant, which leads to

$$
\left\{\begin{array}{l}
T_{t t}(t)-k T(t)=0, \\
a(x) \Phi_{x x}(x)-g \Phi_{x}(x)-k \Phi(x)=0, \quad \Phi(0)=\Phi_{x}(0)=0 .
\end{array}\right.
$$

Solving the second equation of (5.28), we get $\Phi(x)=0$. Therefore, we see that $y(t, x)=T(t) \Phi(x)=0$ for $x \in(0, b)$.

Next, we consider the other subsystem

$$
\begin{cases}y_{t t}(t, x)=\left(\tilde{a}(x) y_{x}(t, x)\right)_{x}, & t>0, x \in(b, 1), \\ M_{1} y_{t t}(t, b)=\tilde{a}\left(b^{+}\right) y_{x}\left(t, b^{+}\right)-a\left(b^{-}\right) y_{x}\left(t, b^{-}\right), & t>0 \\ y(t, b)=0, & t \geq 0 .\end{cases}
$$

From the third equation of (5.29), we have $y_{t}(t, b)=y_{t t}(t, b)=0$. Moreover, noting that $y_{x}\left(t, b^{-}\right)=0$ holds from the above discussion, it follows from the second equation of (5.29) that $\tilde{a}\left(b^{+}\right) y_{x}\left(t, b^{+}\right)=0$, which implies that $y_{x}\left(t, b^{+}\right)=0$ since $\tilde{a}\left(b^{+}\right)>0$. Setting $y(t, x)=T(t) \Phi(x)$, we get $\Phi(b)=\Phi_{x}(b)=0$, and it can be similarly shown that $y(t, x)=T(t) \Phi(x)=0$ for $x \in(b, 1)$.

In this way, we see that $y(t, x)=0$ for $x \in(0,1)$, since $y\left(t, b^{-}\right)=y\left(t, b^{+}\right)$. Consequently, (5.26) holds for any $f(0) \in X$.

REMARK 2. The LaSalle's invariance principle [8, Theorems 3.64 and 3.65] has been often used to show the asymptotical stability in the field of distributed parameter systems. For example, in [7] the invariance principle was used to prove the closed-loop stability with direct strain feedback control law for a flexible robot arm, and, in [4] it was used to prove that a rotating body beam can be stabilized by the torque feedback control law. Recently, the invariance principle was also applied to a force control problem for a constrained one-link flexible manipulator [6].

\section{Numerical Simulation}

Let us set $g=9.8, M_{1}=0.7, M_{2}=1.0$, and $b=0.7$ in system $(2.1)$. We set the initial conditions as $p(x)=0.001\left(e^{5 x}-1\right)+0.1$ and $q(x)=0.0$ for $x \in(0,1)$. Fig. 2 shows the evolution of $y(t, 0.7)$ and $y(t, 1)$ under zero control input, i.e., $u(t) \equiv 0$. The vibration does not decay at all as shown in this figure, since no damping terms are contained in system $(2.1)$ with $u(t) \equiv 0$.

Next, we set the parameters of the control law (3.2) as $\alpha=1.5, \gamma=3.0$, and consider the closed-loop system (4.1). Fig. 3 and Fig. 4 show the evolution of $u(t)$ and the evolution of $y(t, 0.7)$ and $y(t, 1)$, respectively. Fig. 5 shows the evolution of $y(t, x)$ and $y_{t}(t, x)$.

Thus, we see that the control law works effectively for the flexible cable with two rigid loads, although the exponential stability of the closed-loop system is not assured theoretically at this stage. In this numerical simulation, we used MATLAB. 


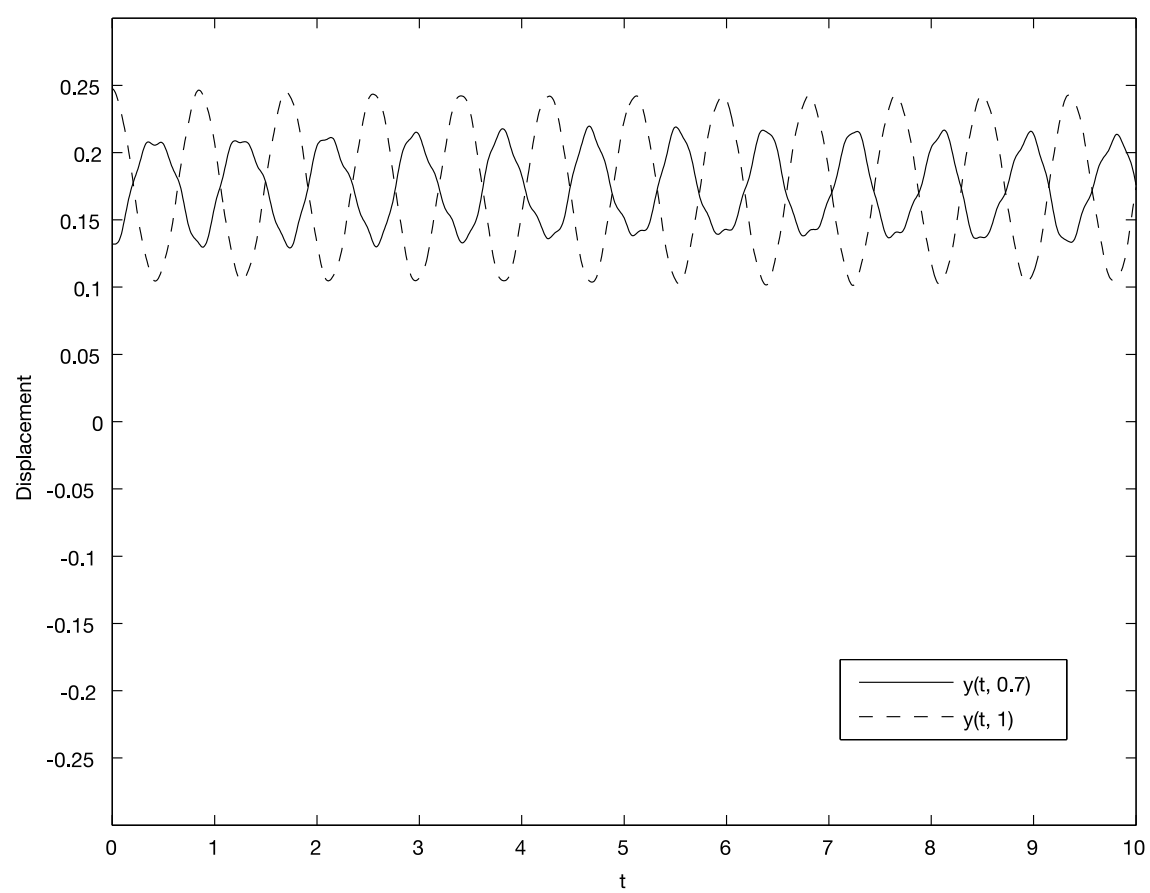

Fig. 2. $y(t, 0.7)$ and $y(t, 1)$ under zero control input.

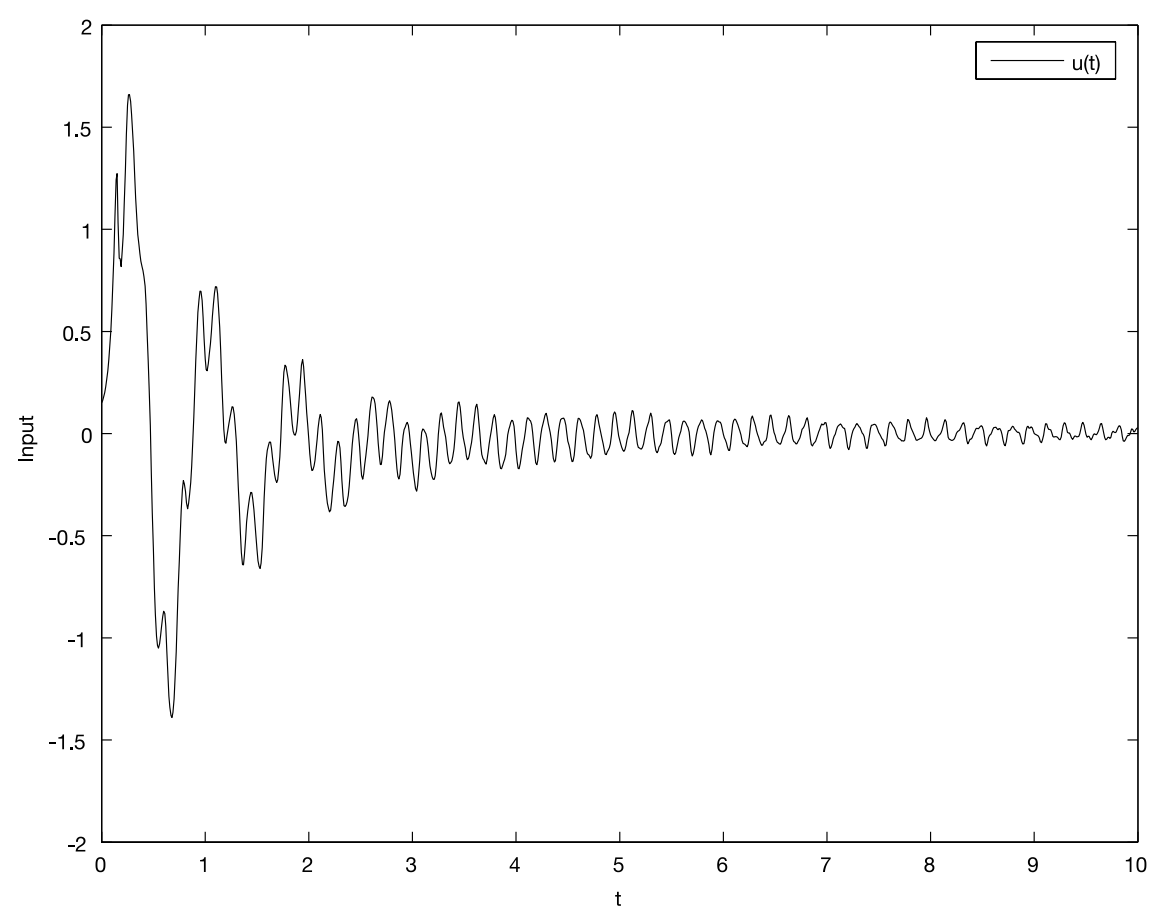

Fig. 3. Control input $u(t)$. 


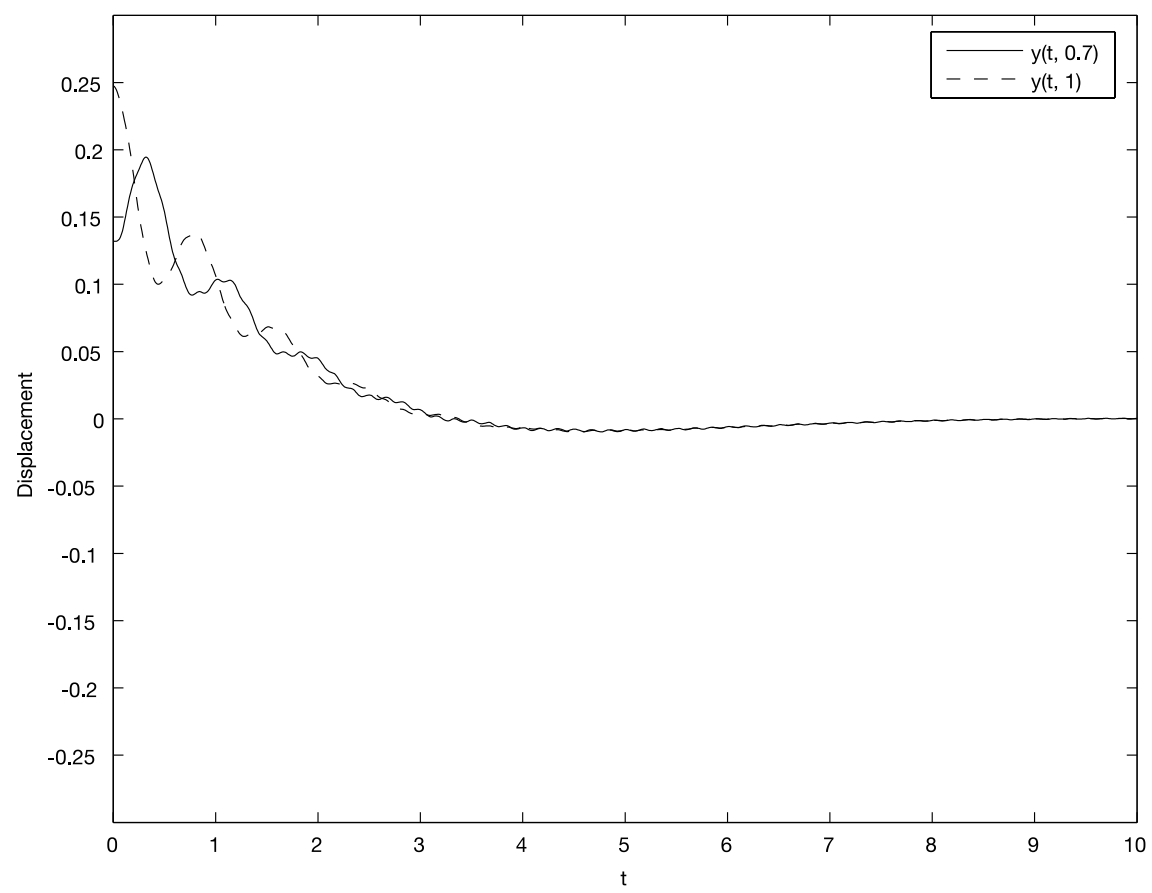

Fig. 4. $y(t, 0.7)$ and $y(t, 1)$ under the control law.
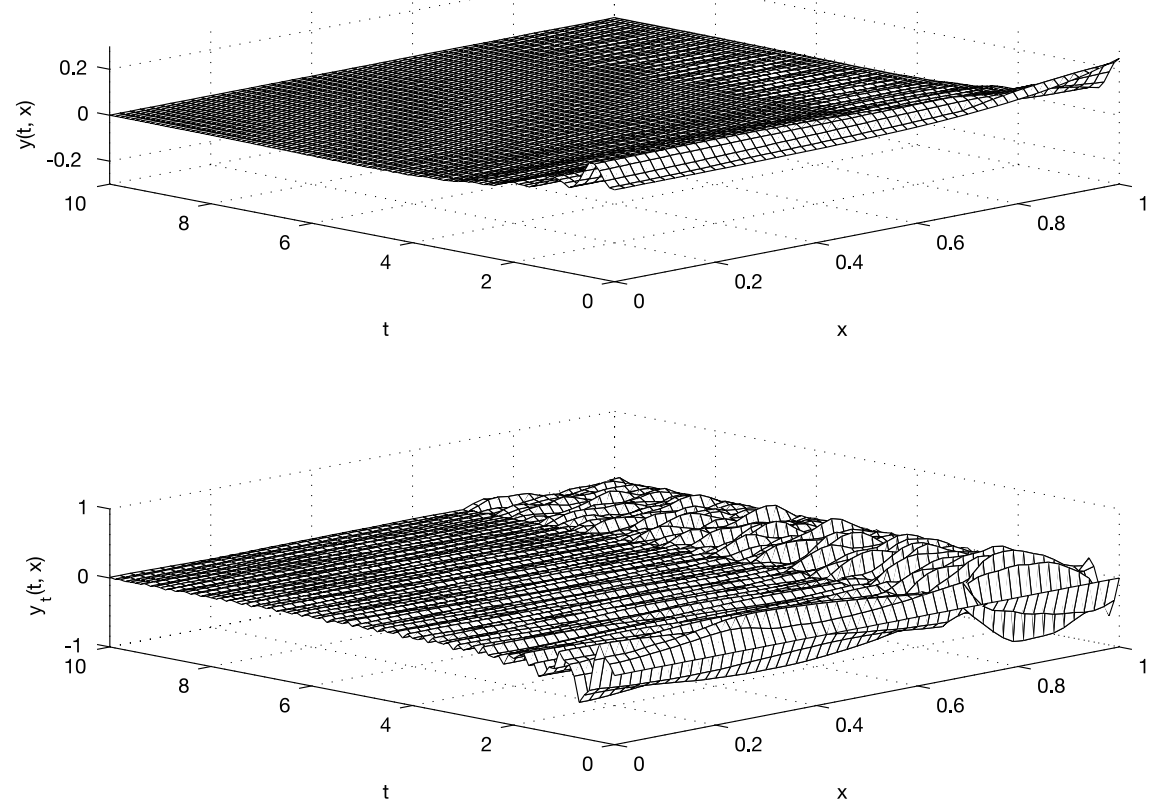

Fig. 5. Evolution of $y(t, x)$ and $y_{t}(t, x)$ under the control law. 


\section{References}

[1 ] K. Ammari, M. Jellouli and M. Khenissi, Stabilization of generic trees of strings. Journal of Dynamical and Control Systems, 11 (2005), 177-193.

[ 2 ] B. d'Andréa-Novel and J.-M. Coron, Exponential stabilization of an overhead crane with flexible cable via a back-stepping approach. Automatica, 36 (2000), 587-593.

[ 3 ] L. Berrahmoune, Pointwise stabilization of strings with non-linear feedback. Mathematical Methods in the Applied Sciences, 27 (2004), 1385-1398.

[ 4 ] J.-M. Coron and B. d'Andréa-Novel, Stabilization of a rotating body beam without damping. IEEE Transactions on Automatic Control, 43 (1998), 608-618.

[5] R.F. Curtain and H.J. Zwart, An Introduction to Infinite-Dimensional Linear Systems Theory. Texts in Applied Mathematics 21, Springer-Verlag, New York, 1995.

[ 6 ] T. Endo and F. Matsuno, Dynamics based force control for an one-link flexible manipulator. Transactions of the Institute of Systems, Control and Information Engineers, 17 (2004), 498-505 (in Japanese).

[ 7 ] Z.H. Luo, Direct strain feedback control of flexible robot arms: new theoretical and experimental results. IEEE Transactions on Automatic Control, 38 (1993), 1610-1622.

[ 8 ] Z.H. Luo, B.Z. Guo and O. Morgul, Stability and Stabilization of Infinite Dimensional Systems with Applications. Springer-Verlag, London, 1999.

[ 9 ] A. Pazy, Semigroups of Linear Operators and Applications to Partial Differential Equations. Applied Mathematical Sciences 44, Springer-Verlag, New York, 1983.

[10] B. Rao, Exponential stabilization of a hybrid system by dissipative boundary damping. Proceedings of the Second European Control Conference, Groningen, 1993, 314-317. 